\title{
EFECTO DEL PICADO SOBRE LAS CARACTERÍSTICAS NUTRICIONALES Y FERMENTATIVAS DE ENSILAJES DE PASTOS KIKUYO, RYEGRASS PERENNE Y ALPISTE FORRAJERO
}

\author{
Luis Villalobos ${ }^{1 / *}$, Jose Arce ${ }^{*}$ \\ Palabras clave: Ensilaje; pastos de clima templado; tamaño de partícula; FDN; aditivos. \\ Keywords: Silage; cool-season grasses; chop length; NDF; additives.
}

Recibido: 06/04/15

Aceptado: $27 / 08 / 15$

\begin{abstract}
RESUMEN
Se evaluó el efecto del picado sobre las características nutricionales y fermentativas de ensilajes elaborados con 3 pastos (kikuyo, ryegrass perenne y alpiste forrajero). Se elaboraron 2 microsilos de cada pasto en bolsas de polietileno con capacidad para $1 \mathrm{~kg}$, aplicando 2 tratamientos (picado y sin picar); se adicionó a cada microsilo $3 \%$ de melaza y $0,1 \%$ de un inóculo artesanal (hecho de leche agria, suero de queso y melaza). La especie de pasto tuvo el mayor impacto sobre las características nutricionales y fermentativas de los microsilos, mientras que el picado no mostró tendencia clara sobre los parámetros evaluados. El contenido de PC $(14,40$ a $19,35 \%), \operatorname{FDN}(46,25$ a $63,16 \%)$ y la DIVMS (46,15 a $65,55 \%)$ en los ensilados es indicador de su potencial para llenar gran parte de los requerimientos nutricionales del ganado lechero. El pasto ryegrass ensilado alcanzó los niveles de $\mathrm{pH}(4,41$ a 4,83) menores y el pasto kikuyo los valores menores de capacidad amortiguadora (79,12 a $81,17 \mathrm{mEq} \mathrm{NaOH} 100 \mathrm{~g}^{-1}$ MS) y de nitrógeno amoniacal (1,03 a 1,33\%). Los ensilajes elaborados con pastos pueden utilizarse como suplementos con valor nutricional superior a los henos utilizados en las raciones de ganado lechero en zonas altas.
\end{abstract}

1 Autor para correspondencia. Correo electrónico: luis.villalobosvillalobos@ucr.ac.cr

\section{ABSTRACT}

Effect of chopping on the nutritional and fermentative characteristics of kikuyu, perennial ryegrass and reed canary grass based silages. The effect of chopping on the nutritional and fermentative traits of 3 grasses (kikuyu, perennial ryegrass and reed canary grass) was evaluated. Two microsilos of each pasture were elaborated in polyethylene bags of $1 \mathrm{~kg}$ capacity, and 2 treatments were applied (chopped or non-chopped). Each microsilo received 3\% (fresh weight) of sugarcane molasses and $0.1 \%$ of a farm-made inoculum (made of sour milk, whey, and molasses). Grass species was the overriding factor on the nutritional and fermentative traits of the microsilos, while chopping showed no clear trend on the parameters evaluated. The contents of CP (14.40 to $19.35 \%)$, NDF (46.25 to 63.16\%), and IVDMD (46.15 to $65.55 \%$ ) in the silages denotes their potential to meet a large share of the nutritional requirements of dairy cattle. Ensiled perennial ryegrass reached the lowest $\mathrm{pH}$ levels (4.41 to 4.83) and kikuyu grass had the lowest values for buffer capacity (79.12 to $81.17 \mathrm{mEq} \mathrm{NaOH} 100 \mathrm{~g}^{-1} \mathrm{DM}$ ) and ammonia nitrogen (1.03 to $1.33 \%$ ). Grass-based silages can be used as supplements with a higher nutritious value than hays used in rations for dairy cattle in highelevation regions.

\footnotetext{
Universidad de Costa Rica, Centro de Investigaciones en Nutrición Animal y Escuela de Zootecnia, Costa Rica.
} 


\section{INTRODUCCIÓN}

La producción láctea en Costa Rica se basa en sistemas en pastoreo que comprenden una gran cantidad de microclimas (Holdridge 1978), esto hace que la productividad de las pasturas varíe durante el año y se presenten épocas de déficit en el suministro de materia seca (MS) al ganado lechero. Asimismo, existen épocas de sobreproducción en las pasturas cuya biomasa puede conservarse por medio de la técnica del ensilaje. El ensilaje es una técnica de conservación de forrajes verdes por medio de la cual se puede suplir la demanda de materia seca del ganado lechero durante las épocas en que el pastoreo se ve limitado por efecto del clima.

La adquisición de pacas de heno o silopacas (henilaje) incrementa el costo de alimentación del ganado lechero en regiones altas de Costa Rica. La elaboración de ensilaje con pastos producidos en finca puede disminuir los costos de suplementación al proveer una fuente de fibra efectiva (Cruz y Sánchez 2000) con un valor nutricional superior al del heno y henilaje (Lindqvist et ál. 2012). El NRC (2001) de ganado lechero recomienda suplir un mínimo de $25 \%$ de la materia seca en forma de fibra detergente neutro, de la cual $75 \%$ debe provenir de forrajes.

El tamaño de partícula del material a ensilar debe estimular el proceso de rumia (Soita et ál. 2000) y permitir una adecuada compactación en el silo. De acuerdo con investigación previa realizada en sistemas de ración totalmente mezclada en Estados Unidos, al menos el 80 a $92 \%$ de las partículas del forraje a ensilar (maíz o alfalfa) deben tener un tamaño menor a 1,9 cm (Jones et ál. 2004). Dicha recomendación pretende optimizar la mezcla del ensilado dentro de la ración para evitar la preferencia y consumo selectivo de los ingredientes más palatables, sin embargo, no garantiza un adecuado proceso fermentativo en el ensilaje.

El picado del pasto a ensilar requiere la utilización de mano de obra y maquinaria, 2 de los rubros de mayor importancia en los costos de producción de ensilajes (Villalobos et ál. 2015). La utilización de pastos sin picar permitiría disminuir los costos de producción de ensilajes.
Sin embargo, no se ha investigado sobre la elaboración de ensilajes con pastos producidos en zonas altas (>1600 msnm) del trópico sin picar. Debido a las características de composición nutricional reportadas en pastos de zonas altas (Andrade 2006, Villalobos y Sánchez 2010, Villalobos 2012), la compactación y fermentación podrían ser distintos a los de otros pastos que tradicionalmente se conservan mediante ensilaje. La presente investigación evaluó el efecto de la especie de pasto (kikuyo [Kikuyuocloa clandestina], ryegrass [Lolium perenne L.] y alpiste forrajero [Phalaris arundinacea L.]) y el picado sobre el valor nutricional y los parámetros de fermentación de ensilajes por medio de la técnica de microsilos.

\section{MATERIALES Y MÉTODOS}

\section{Especies de pastos utilizadas para ensilar}

Se utilizaron pastos kikuyo, ryegrass perenne y alpiste forrajero que fueron cosechados en 3 fincas en la zona alta de Cartago (Costa Rica) con edades de cosecha de 90, 60 y 70 días de rebrote, respectivamente. Las fincas donde se obtuvo el pasto lo cosechaban bajo sistemas de corte y acarreo con las edades utilizadas en este estudio. Una vez cosechados, los pastos se mantuvieron dentro de una instalación cerrada por un período de 24 horas (pre-marchitado) con el objetivo de incrementar el contenido de MS del material debido al proceso de respiración de la planta. Asimismo, el pre-marchitado disminuye el riesgo de descomposición aeróbica (putrefacción) (Conaghan et ál. 2012) y la contaminación ambiental debido a los nutrientes que se pierden en los efluentes (Edmunds et ál. 2014). Posterior al pre-marchitado, una mitad del material cosechado de cada pasto fue picado y la otra mitad se mantuvo como planta entera.

Se utilizó la misma picadora en los 3 pastos con el objetivo de uniformizar el tamaño de partícula; dicho criterio se evaluó por medio del Separador de Tamaño de partícula de la Universidad Estatal de Pennsylvania con el procedimiento descrito por Heinrichs (2010). Dicho separador consiste en 2 bandejas con cribas y una bandeja inferior sin criba 
donde se recolecta el material más fino, lo cual permite separar el material en 3 tamaños de partícula.

\section{Elaboración de microsilos}

Se utilizó la técnica experimental de microsilos debido a que facilita la evaluación de las muestras y comparación entre tratamientos, así como una compactación más consistente (Johnson et ál. 2005). Se utilizaron bolsas de polietileno de empaque al vacío con una capacidad de $1 \mathrm{~kg}$ y un grosor de $0,063 \mathrm{~mm}$. Se evaluaron 2 tratamientos: picado (P) y sin picar (SP), con 4 repeticiones por tratamiento $(8$ muestras por cada pasto) para un total de 24 microsilos. Para las 3 especies evaluadas se registró una muestra de pasto picado para el análisis de distribución de partículas por tamaño, con el separador de la Universidad Estatal de Pennsylvania con el fin de determinar la proporción de partículas según su longitud $(>1,905,1,905$ a 0,780 y $<0,780 \mathrm{~cm})$.

Se elaboró un inóculo artesanal a base de leche agria (1 1), suero de queso $(0,51)$ y melaza $(0,51)$, con lo cual se obtuvo un cultivo madre que se dejó reposar por 2 días y del cual se tomó $15 \mathrm{ml}$, se mezcló con $60 \mathrm{ml}$ de melaza y se completó con agua hasta un volumen de 31 . Finalmente, se aplicó en relación con el peso fresco del material $(0,1 \%)$ para todos los microsilos. El inóculo artesanal fue analizado por su contenido de microorganismos del género Lactobacillus en el Laboratorio de Microbiología Agrícola del Centro de Investigaciones Agronómicas de la UCR y se obtuvo un conteo de $8 \times 10^{6}$ unidades formadoras de colonias por mililitro (UFC. $\mathrm{ml}^{-1}$ ).

Independientemente del tratamiento experimental, todos los microsilos fueron elaborados con melaza al $3 \%(\mathrm{p} / \mathrm{p})$ del peso fresco del pasto e inóculo artesanal en la dosis indicada anteriormente, para luego extraer el aire por medio de una bomba de vacío. Todas las bolsas fueron selladas para prevenir la entrada de aire, se almacenaron a la sombra durante 60 días bajo condiciones de temperatura y humedad ambiental. Finalmente, cada una de las bolsas se abrió y se tomó 2 muestras representativas del material para análisis nutricionales y fermentativos.

\section{Calidad nutricional}

Los análisis nutricionales realizados en los ensilajes fueron materia seca (MS) y proteína cruda (PC) (AOAC 1990), las fracciones de la pared celular (FDN, FDA, y lignina) y la digestibilidad in vitro de la materia seca (DIVMS) (Van Soest y Robertson 1985). Se realizó además los análisis nutricionales a una muestra de cada pasto en el momento de la cosecha para conocer sus características previo al proceso de ensilaje (Cuadros 1 y 2).

Cuadro 1. Contenido nutricional de pastos kikuyo, ryegrass y alpiste en el momento de la cosecha.

\begin{tabular}{lcc}
\hline Pasto $^{1}$ & Materia seca (\%) & Proteína cruda \\
\hline Kikuyo (90 d) & 20,70 & 8,70 \\
Ryegrass perenne (60 d) & 16,50 & 16,97 \\
Alpiste forrajero (70 d) & 16,68 & 17,14 \\
\hline
\end{tabular}

${ }^{1}$ Edad de cosecha de los pastos entre paréntesis.

Cuadro 2. Componentes de la pared celular y digestibilidad de los pastos kikuyo, ryegrass y alpiste en el momento de la cosecha.

\begin{tabular}{lcccc}
\hline Tratamiento $^{1}$ & FDN $(\%)$ & FDA $(\%)$ & Lignina (\%) & DIVMS (\%) \\
\hline Kikuyo (90 d) & 64,20 & 33,40 & 5,20 & 53,90 \\
Ryegrass perenne (60 d) & 53,94 & 34,55 & 3,03 & 66,60 \\
Alpiste forrajero (70 d) & 55,70 & 30,40 & 1,70 & 62,30 \\
\hline
\end{tabular}

${ }^{1}$ Edad de cosecha de los pastos entre paréntesis. 


\section{Parámetros de fermentación}

Los análisis fermentativos realizados en los ensilajes consistieron en medición del pH por medio de un electrodo, la capacidad amortiguadora (CA) (McDonald y Henderson 1962) y el nitrógeno amoniacal (como porcentaje del nitrógeno total) del material después de ensilar (AOAC 1990). Se midió además el pH y la capacidad amortiguadora a una muestra de cada pasto en el momento de la cosecha (Cuadro 3).

Cuadro 3. Variables fermentativas de los pastos kikuyo, ryegrass y alpiste en el momento de la cosecha.

\begin{tabular}{lcc}
\hline Tratamiento $^{1}$ & $\mathrm{pH}$ & Capacidad amortiguadora $\left(\mathrm{mEq} \mathrm{NaOH} 100 \mathrm{~g}^{-1} \mathrm{MS}\right)$ \\
\hline Kikuyo $(90 \mathrm{~d})$ & 8,02 & 6,76 \\
Ryegrass perenne $(60 \mathrm{~d})$ & 7,00 & 273,70 \\
Alpiste forrajero $(70 \mathrm{~d})$ & 9,40 & 66,90 \\
\hline
\end{tabular}

${ }^{1}$ Edad de cosecha de los pastos entre paréntesis.

\section{Análisis estadístico}

El diseño utilizado en este estudio fue de parcelas divididas, para ello la especie de pasto fue la parcela principal y el picado la subparcela con 4 repeticiones. Se realizó un análisis de varianza (ANOVA) con la información de las características nutricionales y los parámetros de fermentación de los microsilos mediante el procedimiento PROC MIXED del paquete estadístico SAS/STAT ${ }^{\circledR}$ 9.3. Asimismo, se obtuvo los cuadrados mínimos para cada una de las combinaciones de tratamientos mediante el siguiente modelo:

Variable analizada $=\mu+\mathrm{P}_{\mathrm{i}}+\mathrm{T}_{\mathrm{j}}+\mathrm{P} * \mathrm{~T}+\mathrm{e}_{\mathrm{ijkl}}$ Donde $\mu$ es la media de las variables de valor nutricional y fermentativo.

$\mathrm{P}$ el i-ésimo efecto de la especie de pasto $(\mathrm{K}, \mathrm{R}, \mathrm{P})$. $\mathrm{T}$ el j-ésimo efecto del picado (P, SP).

$\mathrm{P} * \mathrm{~T}$ el efecto de la interacción pasto por picado.

Se aplicó la prueba de Waller-Duncan para separación de medias en las 6 combinaciones de pastos y tratamientos (KP, KSP, RP, RSP, PP, PSP). Los efectos se consideraron significativos con $\mathrm{p}<0,05$ con base en el error tipo III mediante el procedimiento PROC GLM de SAS/ STAT® 9.3.

\section{RESULTADOS Y DISCUSIÓN}

Posterior al picado, la distribución del tamaño de partícula promedio para los 3 pastos evaluados fue $89,26,8,76$ y $1,98 \%$ de las partículas distribuidas en las cribas de $>1,905,1,905$ a 0,780 y $<0,780 \mathrm{~cm}$, respectivamente. La mayor proporción de material se encontró en la criba superior, lo cual pudo deberse al tipo de picadora utilizada pues las cuchillas de disco desmenuzan el pasto sin disminuir su tamaño de partícula. Sin embargo, para fines del estudio la reducción en el tamaño de partícula fue adecuada para permitir la comparación con respecto a la planta entera, así como para modificar la facilidad de compactación del material en las bolsas.

\section{Calidad nutricional}

\section{Materia seca (MS)}

El contenido materia seca en el momento de la cosecha fue mayor en el pasto kikuyo con respecto al ryegrass y alpiste, esto pudo deberse a que el primero se cosechó con más días de rebrote lo que permitió una acumulación mayor de MS (Cuadro 1). La interacción significativa $(\mathrm{p}<0,05)$ entre la especie de pasto y el tratamiento de picado (Cuadro 4), se debió principalmente a las 
Cuadro 4. Contenido nutricional de los ensilados picados y sin picado.

\begin{tabular}{lcc}
\hline Tratamiento & Materia seca $(\%)$ & Proteína cruda $(\%)$ \\
\hline Kikuyo picado & $20,90^{\mathrm{b}}$ & 15,07 \\
Kikuyo sin picar & $23,60^{\mathrm{a}}$ & 14,40 \\
Ryegrass perenne picado & $16,00^{\mathrm{d}}$ & 19,06 \\
Ryegrass perenne sin picar & $16,00^{\mathrm{d}}$ & 18,45 \\
Alpiste forrajero picado & $18,50^{\mathrm{c}}$ & 19,01 \\
Alpiste forrajero sin picar & $18,00^{\mathrm{c}}$ & 19,35 \\
Promedio picado & 18,47 & 17,71 \\
Promedio sin picar & 19,20 & 17,40 \\
Media & 18,83 & 17,56 \\
Especie de pasto $(\mathrm{p}<0,05)^{\dagger}$ & $*$ & $\mathrm{~ns}$ \\
Picado & $*$ & $\mathrm{~ns}$ \\
Especie*picado & $*$ & $\mathrm{~ns}$ \\
\hline
\end{tabular}

$†$ Significancia estadística: ns:no significativo, *significativo.

diferencias en el contenido de MS en los microsilos de pasto kikuyo picado y sin picar. Los microsilos de los 3 pastos tuvieron diferentes $(\mathrm{p}<0,05)$ contenidos de MS debido a las diferencias en cada uno al momento de la cosecha (Cuadro 1). Los microsilos de kikuyo picado mostraron un contenido de MS similar al del material en el momento de la cosecha (Cuadro 1) y los microsilos sin picar tuvieron un contenido mayor $(\mathrm{p}<0,05)$ (Cuadro 4). La MS en los microsilos de ryegrass fue similar con respecto al momento de la cosecha y no hubo diferencias $(\mathrm{p}>0,05)$ debidas al picado del pasto. El pasto alpiste tuvo un contenido de MS numéricamente superior con respecto al momento de la cosecha, sin embargo ambos tratamientos tuvieron contenidos similares.

Los microsilos de kikuyo picado tuvieron un contenido de MS menor al de los ensilados sin picar, lo cual coincide con lo mencionado por Soita et ál. (2000) con ensilajes de cebada con 2 tamaños de partícula $(4,68$ y $18,75 \mathrm{~mm})$, donde los de menor tamaño tuvieron $3 \%$ menos de MS en comparación con los de partícula larga. En latitudes templadas, la MS de los ensilajes ha mostrado disminuciones debido a pérdidas de nutrientes a través de los efluentes (Mulligan et ál. 2002, Conaghan et ál. 2012). Lindqvist et ál. (2012) encontraron valores de $27,3 \%$ de MS en microsilos pre-marchitados por 3 horas con un porcentaje de humedad relativa ambiental $(<40 \%)$ menor a lo reportado en la zona alta de Cartago (>80\%) (IMN 2015). Einarson et ál. (2004) evaluaron 2 tamaños de partícula (10 y $19 \mathrm{~mm}$ ) en ensilajes de cebada, con los que se obtuvieron contenidos de MS de 20,4 y 25,0\%, respectivamente. En términos generales, no se consiguió un incremento en el contenido de MS en los microsilos debido al picado tal como se ha encontrado en los estudios previamente mencionados. En el presente estudio, la diferencia en el tamaño de partícula entre los pastos picados y sin picar pudo no ser suficiente para evidenciar cambios significativos en el contenido de MS en los microsilos.

\section{Proteína cruda (PC)}

El contenido de proteína cruda en los pastos ryegrass perenne y alpiste forrajero fue cercano al doble que el pasto kikuyo en el momento de la cosecha (Cuadro 1). Este último se cosechó en un estado más avanzado de crecimiento, lo cual pudo disminuir su contenido (Villalobos 2012), con respecto a lo reportado para pasturas de kikuyo (Andrade 2006).

El contenido de PC en los microsilos de los 3 pastos fue similar $(\mathrm{p}>0,05)$ y no hubo diferencias entre cada especie de pasto por efecto del picado (Cuadro 4). Soita et ál. (2000) evaluaron 2 tamaños de partícula en ensilajes de cebada y 
obtuvieron contenidos de PC similares (pequeño: 9,9 y largo: $10,3 \%$ PC), lo cual coincide con el presente estudio donde el picado no modificó dicha variable. Los 3 pastos mostraron un contenido de PC mayor en el material ensilado que al momento de la cosecha lo cual podría explicarse por el aporte de nutrientes del inóculo artesanal. En especial para el pasto kikuyo, la concentración de PC mayor en el ensilado fue beneficiosa pues su contenido previo al proceso de ensilaje (Cuadro 1) podría haber limitado la producción de proteína microbial en rumen (NRC 2001).

El contenido proteico de los microsilos fue superior al de silos de maíz (Keady et ál. 2008, Mulligan et ál. 2002) y similar al de silos de mezclas pasto-leguminosa (Lindqvist et ál. 2012). Si se brindan fuentes de carbohidratos fácilmente fermentables (Edmunds et ál. 2014), el $\mathrm{N}$ suplido por el ensilaje de pastos puede ser incorporado y utilizado en forma de proteína microbial ruminal (Conaghan et ál. 2012). El contenido de PC en todos los microsilos fue mayor al de las pacas de heno de transvala (Digitaria decumbens Stent., cv. Transvala) utilizadas normalmente en fincas lecheras de zonas altas (WingChing y Alvarado 2009).

\section{Fibra detergente neutro (FDN)}

El contenido de FDN fue mayor en el pasto kikuyo al momento de la cosecha y similar en los pastos ryegrass y alpiste (Cuadro 2). Para el material ensilado, la interacción entre la especie de pasto y el tratamiento de picado fue significativa $(\mathrm{p}<0,05)$, al ser la especie de pasto con el factor de mayor importancia sobre el contenido de FDN (Cuadro 5). El contenido de FDN fue mayor $(\mathrm{p}<0,05)$ para los microsilos de pasto kikuyo, mientras que el ryegrass y el alpiste tuvieron valores similares entre ellos. El pasto kikuyo utilizado para elaborar los microsilos tuvo una edad de rebrote mayor con respecto al ryegrass y alpiste, asimismo, estos 2 pastos son especies de clima templado $\left(\mathrm{C}_{3}\right)$ cuyo contenido de pared celular tiende a ser menor (Sage y Kubien 2007) por lo tanto, a pesar de que dichos factores no fueron evaluados en este experimento, podrían haber influido en los resultados observados. Adicionalmente, todos los tratamientos tuvieron un contenido de FDN menor al reportado por WingChing y Alvarado (2009) en pacas de heno de transvala, las cuales son una de las fuentes de suplementación comunes en fincas lecheras de altura.

Cuadro 5. Componentes de la pared celular y digestibilidad de los ensilados picados y sin picado.

\begin{tabular}{|c|c|c|c|c|}
\hline Tratamiento & FDN $(\%)$ & FDA $(\%)$ & Lignina (\%) & DIVMS (\%) \\
\hline Kikuyo picado & $63,16^{\mathrm{a}}$ & $33,73^{\mathrm{a}}$ & $3,59^{\mathrm{a}}$ & $46,15^{\mathrm{e}}$ \\
\hline Kikuyo sin picar & $61,87^{\mathrm{a}}$ & $33,05^{\mathrm{ab}}$ & $4,02^{\mathrm{a}}$ & $48,35^{\mathrm{e}}$ \\
\hline Ryegrass perenne picado & $53,12^{\mathrm{b}}$ & $28,75^{\mathrm{c}}$ & $2,50^{\mathrm{b}}$ & $61,15^{\mathrm{b}}$ \\
\hline Ryegrass perenne sin picar & $46,25^{\mathrm{c}}$ & $27,50^{\mathrm{c}}$ & $1,87^{\mathrm{b}}$ & $65,55^{\mathrm{a}}$ \\
\hline Alpiste forrajero picado & $49,74^{\mathrm{bc}}$ & $32,46^{\mathrm{ab}}$ & $3,77^{\mathrm{a}}$ & $52,90^{\mathrm{d}}$ \\
\hline Alpiste forrajero sin picar & $53,34^{\mathrm{b}}$ & $32,20^{\mathrm{b}}$ & $4,55^{\mathrm{a}}$ & $56,85^{\mathrm{c}}$ \\
\hline Promedio picado & 55,34 & 31,65 & 3,29 & 53,40 \\
\hline Promedio sin picar & 53,82 & 30,91 & 3,18 & 56,92 \\
\hline Media & 54,58 & 31,28 & 3,24 & 55,16 \\
\hline Especie de pasto $(\mathrm{p}<0,05)^{\dagger}$ & $*$ & $* *$ & $*$ & $*$ \\
\hline Picado & ns & $\mathrm{ns}$ & $*$ & $*$ \\
\hline Especie* picado & $*$ & $*$ & $*$ & $\mathrm{~ns}$ \\
\hline
\end{tabular}

$†$ Significancia estadística: ns: no significativo, *significativo, **altamente significativo. 


\section{Fibra detergente ácida (FDA)}

El contenido de FDA fue similar en los pastos kikuyo y ryegrass y menor en el pasto alpiste al momento de la cosecha (Cuadro 2). Con respecto a los análisis pos fermentación, la interacción entre la especie de pasto y el tratamiento $(\mathrm{p}<0,05)$ se debió principalmente a las diferencias en el contenido de FDA en cada pasto ( $p<0,0001)$ (Cuadro 5). El picado no produjo diferencias en el contenido de FDA entre cada especie de pasto, al mostrar los microsilos de pastos kikuyo y alpiste valores similares y el ryegrass valores menores $(\mathrm{p}<0,05)$. Einarson et ál. (2004) mencionan que la FDN y la FDA, normalmente, no se ven afectadas por efecto del picado en ensilajes. Los mismos autores indican que se requiere de una diferencia amplia en el tamaño de partícula para que ambos componentes de la pared celular se vean modificados durante el proceso de ensilaje. Es posible que la diferencia en el tamaño de partícula entre los microsilos picado y sin picar no fue suficiente para producir un cambio significativo en el contenido de la FDA.

\section{Lignina}

El pasto kikuyo utilizado en los microsilos presentó una edad de cosecha mayor, lo cual podría haber influido en un contenido de lignina mayor con respecto a los otros 2 pastos (Cuadro 2). La interacción entre la especie de pasto y el picado se vio afectada principalmente por las diferencias encontradas entre los ensilados de ryegrass con respecto a los de alpiste y kikuyo (Cuadro 5).

\section{Digestibilidad in-vitro de la materia seca (DIVMS)}

La digestibilidad del pasto kikuyo en el momento de la cosecha fue menor a la del ryegrass y alpiste (Cuadro 2), lo cual coincide con el contenido mayor encontrado en los componentes de la pared celular. La digestibilidad de los microsilos mostró diferencias debidas a los efectos individuales de la especie de pasto y el picado (Cuadro 5). De igual forma que en el momento de la cosecha, el pasto ryegrass perenne tuvo digestibilidades mayores $(\mathrm{p}<0,05)$ que los pastos alpiste y kikuyo. Los microsilos sin picar de pastos alpiste y ryegrass tuvieron digestibilidades mayores con respecto a los picados. $\mathrm{La}$ DIVMS fue menor en los ensilados picados de ryegrass y alpiste con respecto a los ensilados de dichas especies sin picar. Ambos pastos tuvieron un contenido de humedad mayor lo cual pudo producir pérdidas de nutrientes solubles debido a la ruptura de la pared celular. Por otra parte, los microsilos de kikuyo mostraron valores similares $(p>0,05)$ de DIVMS para ambos tratamientos. En sistemas intensivos de pastoreo, corte y acarreo, la cosecha se realiza cuando el pasto se encuentra en un estado de crecimiento vegetativo (menos días de rebrote) por lo que las digestibilidades son normalmente mayores (Andrade 2006, Villalobos y Sánchez 2010, Villalobos 2012) que las obtenidas en el presente estudio.

Un mes de diferencia en la edad de cosecha ha mostrado diferencias significativas $(\mathrm{p} \leq 0,0001)$ en la digestibilidad (64,70 a 77,60\% DIVMS) de ensilajes de pastos de clima templado (Keady et ál. 2008). En el presente estudio, los microsilos de pasto ryegrass tuvieron digestibilidades de casi 20 puntos porcentuales más que los microsilos de kikuyo (Cuadro 5). La elaboración de ensilajes a base de pastos de piso debe tomar en cuenta la edad de cosecha óptima de la especie utilizada (pastos tropicales vs pastos de clima templado) que permita obtener la mayor cantidad de materia seca con un alto valor nutricional. Con excepción de los microsilos de kikuyo picado, todos los tratamientos tuvieron digestibilidades mayores a las reportadas en heno de transvala (WingChing y Alvarado 2009). El ensilaje de pastos de piso tiene potencial de proveer materia seca con una calidad superior a la que proveen fuentes de suplementación de fibra larga como el heno de transvala y que se utilizan en épocas de déficit en el consumo de MS en ganado lechero.

\section{Parámetros de fermentación}

\section{pH y Capacidad amortiguadora (CA)}

El pH de los pastos en el momento de la cosecha fue alcalino en los pastos kikuyo 
y alpiste y neutro para el ryegrass (Cuadro 3). La especie de pasto $(\mathrm{p} \leq 0,0001)$ fue el factor de mayor influencia sobre la interacción con el tratamiento de picado $(\mathrm{p}<0,05)$ (Cuadro 6). Los microsilos de ryegrass perenne tuvieron los valores de $\mathrm{pH}$ menores seguidos por el pasto kikuyo y el alpiste. No hubo una tendencia clara por efecto del picado en cada especie de pasto, así los microsilos de pasto kikuyo picado tuvieron un $\mathrm{pH}$ mayor $(\mathrm{p}<0,05)$ que los de pasto sin picar. En el pasto ryegrass los microsilos picados tuvieron un $\mathrm{pH}$ menor y los microsilos de pasto alpiste no fueron diferentes $(\mathrm{p}>0,05)$ por efecto del picado.

Cuadro 6. Variables fermentativas en los ensilados picados y sin picado.

\begin{tabular}{lccc}
\hline Tratamiento & $\mathrm{pH}$ & $\begin{array}{c}\text { Capacidad amortiguadora } \\
\left(\mathrm{mEq} \mathrm{NaOH} 100 \mathrm{~g}^{-1} \mathrm{MS}\right)\end{array}$ & ${\mathrm{N}-\mathrm{NH}_{3} \cdot \mathrm{N}^{\text {total }}{ }^{-1}(\%)}^{1,33^{\mathrm{d}}}$ \\
\hline Kikuyo picado & $5,67^{\mathrm{b}}$ & $79,12^{\mathrm{e}}$ & $1,03^{\mathrm{d}}$ \\
Kikuyo sin picar & $5,09^{\mathrm{c}}$ & $81,17^{\mathrm{e}}$ & $4,43^{\mathrm{b}}$ \\
Ryegrass perenne picado & $4,41^{\mathrm{e}}$ & $120,89^{\mathrm{d}}$ & $6,44^{\mathrm{a}}$ \\
Ryegrass perenne sin picar & $4,83^{\mathrm{d}}$ & $138,87^{\mathrm{c}}$ & $3,20^{\mathrm{bc}}$ \\
Alpiste forrajero picado & $6,09^{\mathrm{a}}$ & $153,99^{\mathrm{b}}$ & $2,56^{\mathrm{cd}}$ \\
Alpiste forrajero sin picar & $6,08^{\mathrm{a}}$ & $165,42^{\mathrm{a}}$ & 3,00 \\
Promedio picado & 5,44 & 120,77 & 3,28 \\
Promedio sin picar & 5,39 & 131,32 & 3,14 \\
Media & 5,42 & 126,05 & $* *$ \\
Especie de pasto $(\mathrm{p}<0,05)^{\dagger}$ & $* *$ & $* *$ & $\mathrm{~ns}$ \\
Picado & $\mathrm{ns}$ & $*$ & $\mathrm{~ns}$ \\
Especie*picado & $*$ & $*$ & \\
\hline
\end{tabular}

$†$ Significancia estadística: ns: no significativo, *significativo, **altamente significativo.

Valores altos de $\mathrm{pH}$ hacen el ensilaje más susceptible a fermentación secundaria y deterioro aeróbico durante la etapa de apertura (Conaghan et ál. 2012). Bajo este criterio, solo los microsilos elaborados con ryegrass picado alcanzaron la acidez $(4,2$ a 4,5) necesaria para evitar descomposición (Boschini y Elizondo 2003). Los ensilajes elaborados con pastos de piso, sin embargo, tienden a mostrar un pH menos ácido en comparación con los ensilajes de maíz (Mulligan et ál. 2002). Los microsilos de pasto ryegrass tuvieron valores de $\mathrm{pH}$ cercanos a lo encontrado en estudios previos con ryegrass (Conaghan et ál. 2012, Keady et ál. 2008, Mulligan et ál. 2002) y con mezclas de pasto-leguminosa (Lindqvist et ál. 2012).

La capacidad amortiguadora (CA) de los pastos en el momento de la cosecha mostró diferencias amplias (Cuadro 3). La interacción entre la especie de pasto y el tratamiento de picado $(\mathrm{p}<0,05)$ fue afectada principalmente por las diferencias encontradas entre las 3 especies evaluadas ( $\mathrm{p} \leq 0,0001)$ (Cuadro 6). Los microsilos de pasto kikuyo tuvieron valores menores, seguidos por el ryegrass y el pasto alpiste tuvo los valores mayores. Lo anterior indica que el pasto alpiste tuvo una mayor susceptibilidad de descomponerse debido a su $\mathrm{pH}$ y $\mathrm{CA}$ mayores (Cuadro 6). Además, no hubo efecto del picado ( $p>0,05)$ en el pasto kikuyo.

Mulligan et ál. (2002) reportan en ensilajes de ryegrass valores de CA similares (136,5 mEq NaOH $100 \mathrm{~g}^{-1} \mathrm{MS}$ ) a los encontrados en el presente estudio. En pastos de clima templado la fracción de proteína verdadera es típicamente mayor que en pastos tropicales (Villalobos 2012), lo cual tiende a incrementar la CA del material (Keady et ál. 2008). Los microsilos de kikuyo tuvieron valores de $\mathrm{pH}$ mayores a los de ryegrass, 
sin embargo, su contenido de MS fue el más alto de los 3 pastos (Cuadro 4), lo cual pudo favorecer su capacidad fermentativa.

\section{Nitrógeno amoniacal $\left(\mathrm{N}-\mathrm{NH}_{3} \cdot \mathrm{N}\right.$ total $\left.^{-1}\right)$}

El $\mathrm{N}$ amoniacal en los microsilos se vio afectado principalmente por las diferencias entre las especies de pasto $(\mathrm{p} \leq 0,0001)$ mientras que el picado no afectó ( $\mathrm{p}>0,05)$ sus valores en los microsilos (Cuadro 6). Los microsilos de ryegrass perenne y alpiste picado tuvieron contenidos de $\mathrm{N}$ amoniacal mayores a lo recomendado en el momento de apertura para ensilajes de maíz $(<3 \%)$ (Boschini y Elizondo 2003). Einarson et ál. (2004) indican que para pastos de piso el contenido de $\mathrm{N}$ amoniacal debe ser inferior al 10\% (Einarson et ál. 2004), lo cual se cumplió en todos los microsilos evaluados. El contenido de $\mathrm{N}$ amoniacal fue menor al de ensilajes de ryegrass perenne de alto y bajo valor nutricional $(11,6 \mathrm{y}$ 14,0\%) (Keady et ál. 2008) y al de ensilajes de pasto-leguminosa sometidos a pre-marchitado con y sin aditivo microbial (7,22 y 8,33\%) (Lindqvist et ál. 2012). Mulligan et ál. (2002) indican que los ensilajes de pastos tienden a mostrar pérdidas de $\mathrm{N}$ en forma amoniacal mayores debido a que su contenido de PC tiende a ser mayor en comparación con ensilajes de maíz.

\section{CONCLUSIONES Y RECOMENDACIONES}

En este estudio la especie de pasto fue el factor de mayor impacto sobre la calidad nutricional de los microsilos mientras que no fue posible evidenciar cambios significativos en los componentes nutricionales como resultado del picado del pasto. Los 3 pastos evaluados tuvieron contenidos de MS menores al 25\%, por lo cual, es probable que estos pastos de piso requieran un contenido de humedad menor que garantice un proceso de ensilaje adecuado.

Los parámetros de fermentación mostraron diferencias debidas principalmente a la especie de pasto mientras que el picado no produjo alguna tendencia clara. El pasto ryegrass tuvo valores de $\mathrm{pH}$ deseables para el proceso de fermentación anaeróbica mientras que el pasto kikuyo tuvo una capacidad amortiguadora y nitrógeno amoniacal deseables en el momento de apertura de ensilajes.

Con base en el contenido de proteína, los componentes de la pared celular y la digestibilidad encontrados, se considera que los ensilajes elaborados con pastos de zonas altas tienen potencial para cubrir gran parte de los requerimientos del ganado lechero y proveer una fuente de fibra larga de calidad nutricional mayor a la del heno y henilaje. Si bien la edad de cosecha no fue un factor analizado en el estudio, la diferencia en las edades de cosecha entre pastos pudo haber influido en los resultados debido a los cambios estructurales que se dan en estados de crecimiento avanzados en los pastos.

En la presente investigación se demostró a nivel experimental que es posible elaborar ensilajes con pastos de zonas altas sin realizar el picado y a la vez mantener una calidad nutricional similar a la de los microsilos de pasto picados. Sin embargo, la alta proporción de partículas mayores a $1,9 \mathrm{~cm}$ en el pasto picado sugiere la importancia de realizar evaluaciones posteriores en donde el tamaño de partícula se reduzca aún más con el objetivo de lograr un contraste mayor en las condiciones fermentativas con respecto al pasto entero.

Evaluaciones posteriores en silos de mayor tamaño permitirán determinar la calidad de compactación y fermentación del pasto sin picar, así como determinar las posibles implicaciones de dicha práctica en los sistemas de alimentación de las fincas.

\section{AGRADECIMIENTOS}

Los autores agradecen a los productores Rolando Ferreto, Alvaro Coto y Rosa Fernández por las facilidades brindadas para la realización del presente estudio, así como a la Dra. Lidieth Uribe del Laboratorio de Microbiología Agrícola del Centro de Investigaciones Agronómicas por su colaboración en el análisis del inóculo utilizado. 


\section{LITERATURA CITADA}

ANDRADE M. 2006. Evaluación de técnicas de manejo para mejorar la utilización del pasto kikuyo (Pennisetum clandestinum Hochst. ex Chiov) en la producción de ganado lechero en Costa Rica, Universidad de Costa Rica, Ciudad Universitaria Rodrigo Facio.

ASSOCIATION OF OFFICIAL AGRICULTURAL CHEMISTS. 1990. Official methods of analysis. 15 ed. Washington, D.C. 1008 p.

BOSCHINI C., ELIZONDO J. 2003. Curso teórico y práctico de ensilaje de forrajes. 1 ed. Editorial de la Universidad de Costa Rica, San José, Costa Rica. 69 p.

CONAGHAN P., O'KIELY P., O'MARA F.P. 2012. Possibilities of increasing the residual water-soluble carbohydrate concentration and aerobic stability of low dry-matter perennial ryegrass silage through additive and cultivar use. Grass and Forage Science 67:177-198.

CRUZ M., SÁNCHEZ J. 2000. La Fibra en la alimentación del ganado lechero. Nutrición Animal Tropical 6:39-74.

EDMUNDS B., SPIEKERS H., SÜDEKUM K.H., NUSSBAUM H., SCHWARZ F.J., BENNETT R. 2014. Effect of extent and rate of wilting on nitrogen components of grass silage. Grass and Forage Science 69:140-152.

EINARSON M.S., PLAIZIER J.C., WITTENBERG K.M. 2004. Effects of Barley Silage Chop Length on Productivity and Rumen Conditions of Lactating Dairy Cows Fed a Total Mixed Ration. Journal of Dairy Science 87:2987-2996.

HEINRICHS J. 2010. Evaluating particle size of forages and TMRs using the Penn State Particle Size Separator. Dairy and Animal Science, Penn State University. Consultado el 12/03/2015. Disponible en http:// extension.psu.edu/animals/dairy/nutrition/forages/ forage-quality-physical/separator

HOLDRIDGE L. 1978. Ecología basada en zonas de vida. Serie libros y materiales de enseñanza. San José, Costa Rica. IICA. 276 p.

INSTITUTO METEOROLÓGICO NACIONAL (IMN). 2015. Climatología de la región del distrito de Santa Rosa de Oreamuno en base a estaciones meteorológicas cercanas a la zona. Informe Anual. 3 p.

JOHNSON H.E., MERRY R.J., DAVIES D.R., KELL D.B., THEODORU M.K., GRIFFITH G.W. 2005. Vaccum packing: a model system for laboratory-scale silage fermentations. Journal of Applied Microbiology 98:106-113.
JONES C.M., HEINRICHS A.J., ROTH G.W., ISHLER V.A. 2004. From harvest to feed: understanding silage management. The Pennsylvania State University. 34 p.

KEADY T.W.J., KILPATRICK D.J., MAYNE C.S., GORDON F.J. 2008. Effects of replacing grass silage with maize silages, differing in maturity, on performance and potential concentrate sparing effect of dairy cows offered two feed value grass silages. Livestock Science 119:1-11.

LINDQVIST H., NADEAU E., JENSEN S.K. 2012. Alphatocopherol and $\beta$-carotene in legume-grass mixtures as influenced by wilting, ensiling and type of silage additive. Grass and Forage Science 67:119-128.

McDONALD P., HENDERSON A.R. 1962. Buffering capacities of herbage samples as factor of silage. Journal of Science Food and Agriculture 13:395-400.

MULLIGAN F.J., QUIRKE J., RATH M., CAFFREY P.J., O’MARA F.P. 2002. Intake, digestibility, milk production and kinetics of digestion and passage for diets based on maize or grass silage fed to late lactation dairy cows. Livestock Production Science 74:113-124.

NATIONAL RESEARCH COUNCIL (NRC). 2001. Nutrient Requirements of Dairy Cattle. $7^{\text {th }}$ rev. Ed. Washington, D.C. National Academy Press. 381 p.

SAGE R.F., KUBIEN D.S. 2007. The temperature response of $\mathrm{C}_{3}$ and $\mathrm{C}_{4}$ photosynthesis. Plant, Cell and Environment 30:1086-1106.

SOITA H.W., CHRISTENSEN D.A., McKINNON J.J. 2000. Influence of Particle Size on the Effectiveness of the Fiber in Barley Silage. Journal of Dairy Science 83:2295-2300.

VAN SOEST P.J., ROBERTSON J.B. 1985. Analysis of forages and fibrous feeds. Cornell University. Ithaca, New York. 165 p.

VILLALOBOS L. 2012. Fenología, producción y valor nutritivo del pasto alpiste (Phalaris arundinacea) en la zona alta lechera de Costa Rica. Agronomía Costarricense 36:25-37.

VILLALOBOS L., ARCE-CORDERO J., WINGCHINGJONES R. 2015. Costos de producción de ensilados de pastos tropicales en lecherías de Costa Rica. Nutrición Animal Tropical 9(2):27-48.

VILLALOBOS L., SÁNCHEZ J. 2010. Evaluación agronómica y nutricional del pasto ryegrass perenne tetraploide (Lolium perenne) producido en lecherías de las zonas altas de Costa Rica. II. Valor Nutricional. Agronomía Costarricense 34(1):43-52.

WINGCHING R., ALVARADO G. 2009. Valor nutricional del heno de transvala inoculado con el hongo Pleurotus ostreatus sp. Agronomía Costarricense $33: 147-153$. 